\title{
Sensitivity pattern of Salmonella species in different age groups
}

\section{Farzeen Zehra ${ }^{1}$, Syeda Mehak Fatima ${ }^{2}$ \& Fatima Syedain}

1,2Jinnah Sindh Medical University

${ }^{3}$ Dr. Essa Laboratory and Diagnostic Centre

Corresponding author: seasum_leo@hotmail.com

\begin{abstract}
Objective: To determine the current efficacy of antibiotics used in treatment of enteric fever, with respect to age, in a laboratory setting. Study Design: A cross-sectional study. Methodology: A retrospective study was done on 105 specimens that were collected at Dr. Essa Laboratory and Diagnostic Centre from January 2012-December 2013. These specimens were inoculated into blood cultures bottles having Oxoid and incubated. Positive specimens were then cultured on EMB, CLED and chocolate agar. These sub cultures were incubated at $37^{\circ} \mathrm{C}$ for about two days.With AP120E screening was done of suspected colonies. They were then processed. Identified isolates were confirmed by specific agglutination antisera. Kirby-Bauer disc diffusion method was used to confirm anti-microbial suspects by Mueller-Hinton agar (Oxoid), using eleven antibiotics which were Amoxicillin, Ciprofloxacin, Aztreonam, Cefixime, Cefotaxime, Ceftriaxone, Co-trimaxole, Enoxacin , Fosfomycin, Sparfloxacin, and Ofloxacin. Results: The overall sensitivity profile shown by these antibiotics against Salmonellae species is as follow : Amoxicillin (0), Aztreonam (47.6\%), Cefixime (98.1\%), Cefotaxime (98.1\%), Ceftriaxone (97.1\%), Ciprofloxacin (85.7\%), Co-trimoxazole $(64.8 \%)$, Enoxacin $(28.6 \%)$, Fosfomycin $(85.7 \%)$, Ofloxacin $(94.3 \%)$, Sparfloxacin $(30.5 \%)$. Conclusion: Third generation cephalosporins (Cefixime, Cefotaxime, Ceftriaxone) have maximum sensitivity profile among all age groups, rendering them most suitable for empirical treatment.
\end{abstract}

Salmonellae species are the causative agents of one of the mos commonly encountered illness by health professionals in developing countries. It causes enteric fever, around 33 million cases and 500,000 deaths have been documented per year (Gautam; $2002 \&$ Saqib; 2000) Salmonella enterica serotype typhi is the major cause, followed by, Salmonellae enterica serotype paratyphi (Abdullah; 2012, Anjum; 2004, Gautam; 2002, Ishaq; 1990, Pokharel; 2006 \& Saqib; 2000) wherever sanitary conditions are not up to the standard it can be considered endemic, as it spreads through contaminated water. This is especially true for countries like Pakistan where it is a huge burden on the economy of the country and causes an increasing amount of mortality and morbidity. Children are predominantly affected group, mainly because of their unhygienic eating habits. In recent years resistance to classically used drugs for the treatment of enteric fever was reported which made quinolones and cephalosporin the popular choice (Abdullah; 2012), but now emergence of strains resistant to multiple drugs has made matters worse(Kumar;2008, Nagshetty;2009, Zaki;2009 \& Mushtaq;2006) and empirical therapy less beneficial (Abdullah; 2012). So to effectively control this disease it is important for everyone to have access to clean and properly chlorinated water, get vaccinated, and antibiotic treatment should be in accordance to sensitivity pattern of the specific isolate that the lab has reported and if empirical treatment is inevitable due to risk of complications, drugs reported to have maximum sensitivity in recent researches should be prescribed. This establishes the need for frequent evaluation of ever changing pattern of antibiotic sensitivity of Salmonellae species, and also laid the foundation of aims of our studies.

\section{Methodolog}

This Study was done on isolates $(\mathrm{n}=105)$ of S. typhi, S. paratyphi A \& B from blood culture samples which were collected from Dr. Essa Laboratory and Diagnostic Centre in Karachi from Jan 2012 to Dec , 2013 . All those blood samples were included which were seropositive for S. typhi, S .paratyphi A \& B. The samples other than blood, and blood samples negative for S. typhi, S. paratyphi A \& B were excluded. Those samples were also excluded which contained superimposed or co-existent infections like HIV etc. In terms of the blood samples, $5-10 \mathrm{ml}$ were taken from adults and 2-3 $\mathrm{ml}$ from children via venepuncture through sterilized instruments. These samples were then introduced/inoculated directly into blood culture bottles which contained $50 \mathrm{ml}$ brain heart infusion broth (Oxoid) and then incubated at $37^{\circ} \mathrm{C}$ for 7 days. Positive blood specimens were further cultured on EMB (Eosin methylene blue), CLED (cystine lactose electrolyte deficient) and chocolate agar .These subcultures were incubated at $35-37^{\circ} \mathrm{C}$ for $24-48$ hours. Screening was done of suspected colonies with $\mathrm{AP} 12 \mathrm{QE}$, processed by standard procedures and the salmonella species isolates were identified and confirmed by specific agglutination antisera. Antimicrobials suspects were determined by the Kirby Bauer disc diffusion method using Mueller Hinton agar(Oxoid) and Amoxicillin (10ug) ,Aztreonam (30ug), Cefixime(5ug) , Cefotaxime(30ug), Ceftriaxone(30ug) ,Cotrimaxole(25ug), Ciprofloxacin(5ug), Enoxacin(5ug), Fosfomycin(50ug), Sparflovaxin(5ug), and Ofloxacin(5ug) discs.

Sample size was calculated through formula for qualitative variable. The data was collected from the laboratory and then statistically analyzed on SPSS 16.0. Percentage and frequencies were calculated, and compared among different age groups.

\section{Results}

Blood samples that were collected between January 2012 - December 2013 had 105 number of isolates that tested positive for Salmonellae species, the most common infective agent being the S. typhi $\quad(n=72$, $68.5 \%)$. This was followed by S. paratyphi $\mathrm{A}(\mathrm{n}=30,28 \%)$, and then S. paratyphi B $(n=3,2.8 \%)$. Males were slightly more affected $(n=58$, $55.2 \%)$ than females $(\mathrm{n}=47,44.7 \%)$. The most commonly affected population was noted to be children (neonates-12 years) $(n=67,63.8$ $\%)$, young adults (19-45 years) were on second number $(\mathrm{n}=19,18 \%)$ then adolescents (14-18 years) $(n=17,16 \%)$, and comparatively lesser number of people from the adult age range (45-60 years) were noted to be victims of enteric fever $(n=2,1.9 \%)$

Cefixime, Cefotaxime, Ceftriaxone(all three 3rd generation cephalosporin) and Ofloxacin were noted to have the highest efficacy against Salmonellae species, showing little variance with age, having $98.1 \%, 98.1 \%, 97.1 \%$ and $94.3 \%$ overall sensitivity respectively. Aztreonam seemed to have changes in the sensitivity pattern among different age groups with $100 \%$ sensitivity in adults , $47.3 \%$ in young adults, $47.0 \%$ in adolescents and $46.2 \%$ in children (p-value 0.700 ) (Table 2). Similarly ,Fosfomycin too is predominantly sensitive in adult age range showing $100 \%$ sensitivity, $89.14 \%$ in young adults, $82.5 \%$ in adolescents and $85.0 \%$ in children, but here variation with age is not much significant ( $\mathrm{p}$ value 0.777 ) (Table 2). Overall, Enoxacin has sensitivity of just $28.6 \%$, but in adults it has reached up to $100 \%$, with a gradual decline in young adults $(73.6 \%$ ), and adolescents $(70.5 \%)$, and then an abrupt decline in children with just $2.9 \%$ isolates found sensitive to Enoxacin ( p value 0.777) (Table 2). 
Co-trimoxazole also has a sensitivity pattern that varies with age but here isolates taken from adults were predominantly resistant to it , showing $100 \%$ resistance, then rapid decline was seen in young adults, adolescents and children, in whom resistance went down to $31.5 \%, 23.5 \%$ and $34.3 \%$, respectively ( $\mathrm{p}$ value 0.716 ) (Table 2). Salmonellae species are highly resistant against Amoxicillin with 100 $\%$ resistance among all age group.

TABLE 1: Antibiotic sensitivity pattern of Salmonellae species in different age groups

\begin{tabular}{|c|c|c|c|}
\hline Antibiotics & Neonates-13 years & $14-18$ years & $19-45$ years \\
\hline Cefixime & $65(97 \%)$ & $17(100 \%)$ & $19(100 \%)$ \\
\hline Cefotaxime & $65(97 \%)$ & $17(100 \%)$ & $19(100 \%)$ \\
\hline Ceftriaxone & $65(97 \%)$ & $16(94.1 \%)$ & $19(100 \%)$ \\
\hline Aztreonam & $31(46.2 \%)$ & $8(47 \%)$ & $9(47.3 \%)$ \\
\hline Fosfomycin & $57(85.0 \%)$ & $14(82.3 \%)$ & $17(89.4 \%)$ \\
\hline Ofloxacin & $62(92.5 \%)$ & $17(100 \%)$ & $18(94.7 \%)$ \\
\hline Enoxacin & $2(2.9 \%)$ & $12(70.5 \%)$ & $14(73.6 \%)$ \\
\hline Ciprofloxacin & $55(82.0 \%)$ & $15(88.2 \%)$ & $18(94.7 \%)$ \\
\hline Sparflovaxin & $5(7.4 \%)$ & $12(70.5)$ & $13(68.4 \%)$ \\
\hline Co-trimoxazole & $43(64.1 \%)$ & $12(70.5)$ & $2(100 \%)$ \\
\hline Amoxicillin & 0 & 0 & $2(100 \%)$ \\
\hline
\end{tabular}

FIGURE 1: Incidence of Enteric fever in different age groups

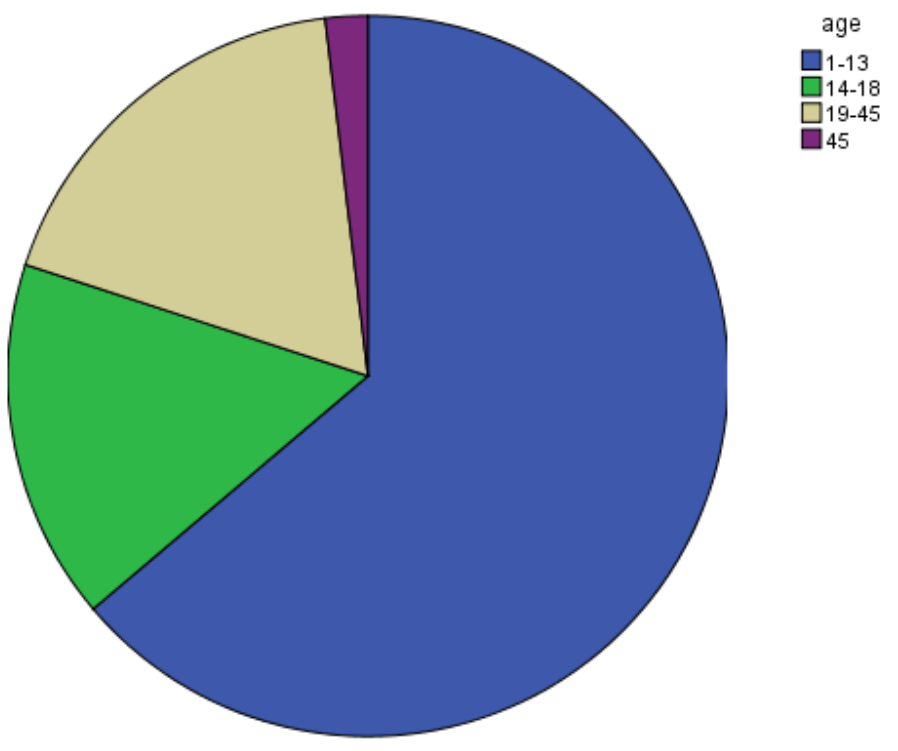

TABLE 2: p-value of antibiotics relative to age

\begin{tabular}{|c|c|}
\hline Antibiotics & p-value \\
\hline Cefixime & 0.006 \\
\hline Cefotaxime & 0.006 \\
\hline Ceftriaxone & 0.111 \\
\hline Aztreonam & 0.700 \\
\hline Fosfomycin & 0.777 \\
\hline Ofloxacin & 0.716 \\
\hline Enoxacin & 0.777 \\
\hline Ciprofloxacin & 0.700 \\
\hline Co-trimoxazole & 0.716 \\
\hline
\end{tabular}

\section{Discussion}

Salmonellae species are the cause of major health problems in Pakistan, transmitted through contaminated food and water they can cause food poisoning and in more severe bacteremia they can result in typhoid fever. If not treated promptly, the complications can be catastrophic resulting in splenomegaly, intestinal perforation and cholecystitis among many others.

Our study indicates that the most vulnerable age group is from neonates to 12 years old; whereas some other suggest that it is $1-10$ years (Abdullah; 2012, Bhan; 2005, Kumar; 2008 \& Saqib; 2000). 
A study conducted in Pakistan quantifies that 20-29 years is the age group that is most susceptible (Hafiz; 1998), which is strongly in contrast with our findings. In another study from New Delhi, children ranging from age 6-15 years made up $42.5 \%$ of the total infected population (Kumar; 2008) . Basically, Karachi like other large cities of the developing world, Mexico City and Santiago have epidemiologic pattern of spread of typhoid disease that is known to majorly affect children and adolescents (Siddiqui; 1991), in this pattern of epidemiology distribution of disease is predominant in areas with poor salutary conditions (Siddiqui; 1991). This explains why children are the most vulnerable group, because probability of bacteria transmission is higher at schools where they eat and drink together in unhygienic conditions and there is very little surveillance on the quality of food sold at school canteens. Additionally, sanitary conditions are inappropriate, making it very difficult to create a germ free environment.

The sensitivity of drugs depends upon different strains of Salmonellae, as well as geographical factors. Also, the origin of the isolate play an important role (Abdullah; 2012).

The studies conducted in Nepal and India, also part of subcontinent, have slightly different results as compared to ours. Fluoroquinolones have the highest sensitivity in Pakistan, whereas Co-trimoxazole has the highest sensitivity in Nepal, than Pakistan and India (Gautam; 2002 \& Pokharel; 2006) various drugs have been used in the management of enteric fever over the years but their effect on Salmonellae has never been the same. Amoxicillin, which was once the first line of treatment, is now observed to have the lowest efficacy in numerous studies (Abdullah; 2012, Gautam; 2002 \& Kumar; 2008), which is also the finding of our research. Also, we evaluated $64.8 \%$ of an overall sensitivity to Cotriamoxazole is evaluated by us, $10 \%$ more than results found in other studies (Abdullah; 2012 \& Gautam; 2002), which showed a $54.8 \%$ amount of sensitivity to Co-trimoxazole.

The third generation cephalosporin like Cefixime, Cefotaxime and Ceftriaxone showed very little variance in their sensitivity with research that was previously done in Essa Laboratory, Karachi from 2008-2010 (Abdullah; 2012), where 96.2\%, 99.17 and $98.79 \%$ sensitivity was respectively documented. In northern part of India from 1997-2001 a study was conducted where Cefotaxime showed $79 \%$ sensitivity, Cefixime $92 \%$ and Ceftriaxone $88 \%$ (Gautam; 2002). Again, diversity from our findings is negligible.

Salmonellae typhi is the major infecting specie out of three, a finding that was consistent with several other studies (Abdullah; 2012, Anjum; 2004, Gautam; 2002, Ishaq; 1990, Pokharel; 2006, Saqib; 2000 \& Tankhiwale; 2003). A study conducted in New Delhi, India reported the ratio of Salmonellae typhi to Salmonellae paratyphi A to be 4:1 (Bhan; 2005), we appraised it to be 3:1.25, emphasizing on the suggestion by some studies that the prevalence of S. paratyphi -A is in fact increasing (Abdullah; 2012, Kumar; 2008 \& Tankhiwale; 2003). Males were affected slightly more than females with a ratio of 1.4:1.8, and this outcome is very similar to what that has been observed is some other published works (Abdullah; 2012, Kumar; 2008). For example, one study reports nearly the same result of $55.7 \%$ affected the male population (Kumar; 2008).

Currently Fluoroquinolones are the most popular drug for Salmonellae infection, prescribed promptly in all age groups, even in children, in whom the use of this drug is contraindicated since it caused connective tissue problem in immature animals during laboratory testing. Despite experiments suggest otherwise, the widespread use of this drug in pediatric medicine indicates the need of strict regulations on drugs that are used in the treatment of typhoid fever. Fluoroquinolones are also contraindicated in pregnant women (Ishaq; 1990). This trend of prescribing Fluoroquinolones empirically, has caused increased resistance to it (Bhan; 2005), and for the first time in 1993 Fluoroquinolone were unable to treat enteric fever in Pakistan (Abdullah; 2012), indicating alteration in DNA gyrase and acquisition of plasmid -mediated resistance. MDR strains have also emerged (Bhan; 2005 \& Nagshetty; 2009), in fact by 1972 it was widespread in India (Kumar; 2008, Mushtaq; 2006 \& Ranju; 1998). Although in Pakistan first case was reported in 19872 (Zaki; 2011),but now a study indicates that prevalence of MDR strains has increased up to $62.64 \%$ (Abdullah;2012). Recent studies from Quetta and Bahawalpur, cities of Pakistan reported a $69 \%$ and 53.8\% prevalence of MDR strains (Mushtaq; 2006), respectively. This should come as no surprise because in our part of the world self-medication and over the counter sale of drugs is considered a norm. Worryingly, it can have disastrous consequences because Fluoroquinolones, just like ciprofloxacin and Ofloxacin, shows poor response to MDR isolates according to studies conducted in Kathmandu Nepal( Pokharel;2006). However, the same study indicates positive response by 3rd generation cephalosporin, implicating their importance in the eradication of infection by MDR strain.

In Pakistan, summer and monsoon seasons are when the highest numbers of cases are reported (Abdullah; 2012), so unsurprisingly most of the cases we studied were reported in this time of the year. It's mainly because microorganisms flourish in heat and moisture. But also, it is because infection is transmitted when bacteria is ingested in high concentrations through food and drink and this occurs more frequently in these seasons because over flowing sewerage lines in cities like Karachi mix with fresh water and reach our taps.

In our study all blood samples that were collected were from residents of Karachi, Pakistan.

\section{Conclusion}

The findings of our research conclude that during childhood the probability of contracting infection by Salmonellae typhi is the highest. During same period physicians can face most difficulty in curing disease and eradicating microorganism because many antibiotics exhibit a decreased efficacy in this age, relative to adults. Third generation Cephalsporins apparently are most suitable for empirical treatment in all ages, including children. Ofloxacin despite its good sensitivity should be avoided in this age due to potential side effects.

\section{Conflict of Interest}

The author declare that there are no conflict of interest.

Acknowledgement

We are grateful to Dr. Farhan Essa Abdullah from Dr. Essa Laboratory and Diagnostic Centre, and Dr. Syed Muhummad Mustahsan for their guidance.

\section{References}

- Abdullah, F. E., Haider, F., Fatima, K., Irfan, S., \& Iqbal, M. S. (2012). Of Salmonellae Isolates. Journal of the College of Physicians and Surgeons Pakistan, 22(3), 147-150.

- Anjum, P., Qureshi, A. H., \& Rafi, S. (2004). Fluroquinolone resistance in typhoidal salmonella and its detection by Nalidixic acid disc diffusion. Journal-Pakistan medical association, 54(6), 295-300.

- Bhan, M. K., Bahl, R., \& Bhatnagar, S. (2005). Typhoid and paratyphoid fever.The Lancet, 366(9487), 749-762.

- Gautam, V., Gupta, N. K., Chaudhary, U., \& Arora, D. R. (2002). Sensitivity pattern of Salmonella serotypes in Northern India. Brazilian journal of infectious diseases, 6(6), 281-287.

- Hafiz, S., Habib, F., Ahmed, N., Haq, I. U., \& Husain, R. (1998). Typhoid fevers: treatment with lomefloxacin. Journal of Pakistan Medical Association,48(6), 168-170 
- Ishaq, M., Farooqui, B. J., Ashfaq, M. K., \& Khan, M. A. (1990). Therapeutic implications of ofloxacin in the treatment of typhoid fever caused by multiply resistant Salmonella typhi. JPMA, 40(8), 176-78.

- Kumar, S., Rizvi, M., \& Berry, N. (2008). Rising prevalence of enteric fever due to multidrug-resistant Salmonella: an epidemiological study. Journal of medical microbiology, 57(10), 1247-1250.

- Mushtaq, M. A. (2006). What after ciprofloxacin and ceftriaxone in treatment of Salmonella typhi. Pakistan Journal of Medical Sciences, 22(1), 51.

- Nagshetty, K., Channappa, S. T., \&Gaddad, S. M. (2009). Antimicrobial susceptibility of Salmonella typhi in India. The Journal of Infection in Developing Countries, 4(02), 070-073.

- Pokharel, B. M., Koirala, J., Dahal, R. K., Mishra, S. K., Khadga, P. K., \&Tuladhar, N. R. (2006). Multidrug-resistant and extended-spectrum beta-lactamase (ESBL)-producing Salmonella enterica (serotypes Typhi and Paratyphi A) from blood isolates in Nepal: surveillance of resistance and a search for newer alternatives. International journal of infectious diseases, 10(6), 434-438.
- Ranju, C., Pais, P., Ravindran, G. D., \& Singh, G. (1998) Changing pattern of antibiotic sensitivity of Salmonella typhi. National Medical Journal of India, 11, 266-267.

- Saqib, A., \& Ahmed, A. (2000). Culture and sensitivity of salmonella species: Analysis of a twoyear data. Journal of the Pakistan Medical Association, 50.

- Siddiqui, S. (1991). Epidemiologic patterns and control strategies in typhoid fever. Journal of the Pakistan Medical Association, 41(6), 143-146.

- Takkar, V. P., Kumar, R., Takkar, R., \&Khurana, S. (1995). Resurgence of chloramphenicol sensitive Salmonella typhi. Indian pediatrics, 32, 586-586.

- Tankhiwale, S. S., Agrawal, G., \&Jalgaonkar, S. V. (2003). An unusually high occurrence of Salmonella enterica serotype Paratyphi A in patients with enteric fever. Indian Journal of Medical Research, 117, 10-12.

- Zaki, S. A., \& Karande, S. (2011). Multidrug-resistant typhoid fever: a review.The Journal of Infection in Developing Countries, 5(05), 324-337. 